\title{
Comments on the Linear Transverse Coupling
}

\section{Introduction}

One of the most troublesome phenomena during the commissioning of synchrotrons is the linear horizontal-vertical coupling. Because of its linear nature, one can in principle obtain the analytical solution provided that all sources of skew quadrupole field are known completely in the entire ring. In spite of this, many standard diagnostic procedures and measurements become rather confusing and of ten lead to wrong conclusions. The purpose of this note is to explain some of the simpler relations which are useful in understanding measurement of tunes and the amount of coupling.

If all sources of skew quadrupole field are known, one can easily calculate the $(4 \times 4)$ transfer matrix at any point around the ring in $\left(x, x^{\prime}=d x / d s, y, y^{\prime}=d y / d s\right)$ phase space and parametrize the matrix such that there are ten independent quantities to specify the coupled motion completely. This convenient parametrization has been proposed by D. Edwards and L. Teng. ${ }^{1}$ of ten parameters, six are needed to specify the uncoupled motion in two eigen-directions ( $u$ and $v$, say) and the remaining four are used to specify the transformation from $(u, v)$ to $(x, y)$. The motion in $\left(x, x^{\prime}\right)$ or in $\left(y, y^{\prime}\right)$ phase space is a mixture of two eigentunes, $v_{1}$ and $v_{2}$, which, in the limit of no coupling, reduce to $\nu_{x}$ and $\nu_{y}$ :

$$
\begin{aligned}
& \nu_{1}+\text { larger of }\left(v_{x}, v_{y}\right) ; \quad v_{1}>v_{2} \\
& v_{2}+\text { smaller of }\left(v_{x}, v_{y}\right)
\end{aligned}
$$


When the coupling is introduced intentionally by exciting known skew quadrupoles, one can follow the Edwards-Teng formalism or Eq. (4.84) of Courant \& Snyder 2 and calculate two eigen-frequencies $\left(v_{1}\right.$ and $\left.v_{2}\right)$. In electron synchrotrons, it is customary to specify the degree of coupling by the "coefficient of coupling" $k$,

$$
\varepsilon_{x}=\frac{\varepsilon_{n}}{I+k}, \quad \varepsilon_{y}=\frac{k \varepsilon_{n}}{I+k}
$$

where $\varepsilon_{n}=$ natural emittance determined by the quantum excitation and radiation damping; $\varepsilon_{x}, \varepsilon_{y}=$ horizontal and vertical emittances which are related to the rms beam sizes $\sigma_{x}=\sqrt{\beta_{x}^{\varepsilon} x}$ and $\sigma_{y}=\sqrt{\beta_{y}^{\varepsilon} y}$. A small but finite amount of coupling is usually required in electron synchrotrons in order to prevent beam instabilities arising from $\sigma_{y} \rightarrow 0$. In proton synchrotrons, one almost always tries to minimize the amount of coupling.

A difficulty one must face during the commissioning (and even later) is that the sources of linear coupling around the ring cannot be known completely since the coupling is generated by the random rolls of quadrupole magnets and the vertical closed-orbit distortions in sextupole magnets. Effective field gradients of skew quadrupole field are given by

$$
B^{\prime} S Q=-2{ }^{\theta} R B^{\prime} Q \text { or } \quad B^{\prime} S Q=B^{\prime \prime} y_{C}
$$

where $\theta_{R}=$ roll angle of quadrupole with gradient $B_{Q}$ and $y_{C}=$ vertical closed orbit in sextupole with strength B".

Under such circumstances, one must define a measurable quantity which represents the amount of coupling so that it can be, for example, simply related to the parameter $k$. Since this quantity is to represent the combined 


\section{DISCLAIMER}

Portions of this document may be illegible in electronic image products. Images are produced from the best available original document. 
effects of many sources, a certain condition must exist in order for this representation to be useful and meaningful. The condition is that, of all Fourier components of the skew field around the ring, only one component is important in describing the coupled motion. This is often referred to as the "single or isolated resonance" approximation.

In the absence of linear coupling, assume that two tunes are $v_{x}$ and $\nu_{y}$ (which are yet to be found by some measurements). The Hamiltonian for the coupled motion is composed of infinite number of oscillating terms of the form

$$
\exp i\left(\nu_{x} \pm \nu_{y}+n\right) \theta
$$

where $\theta=s / R$ ( $R=$ average machine radius, $s=$ path length) is the azimuthal angle. Near $v_{x} \pm \nu_{y}=N$ (integer) $+\Delta$ with $|\Delta| \ll 1$, one argues that the only component to be retained is the one with the lowest frequency of oscillation, i.e., the one with $n=-N$. All other terms oscillate so rapidly that their average effects over many revolutions can be ignored. The quantity which is used to represent the combined effects of all sources of skew quadrupole field is

$$
C_{N}=(1 / 4 \pi) \Sigma\left(B^{\prime} S Q \ell / B \rho\right) \sqrt{\beta_{x}^{B} y} \exp i\left(\theta_{x} \pm \theta_{y}+N \theta\right)
$$

where

$$
\theta_{x, y}=\psi_{x, y}-\nu_{x, y} y^{\theta}=\int_{0}^{s}\left(d s / \beta_{x, y}\right)-\nu_{x, y^{\theta}}
$$

are to be defined in the absence of coupling; otherwise, quantities such as ${ }^{B} x, y$ and $v_{x, y}$ are meaningless. Note that $C_{N}$ is in general a complex number and the coupling is represented by just two real parameters. Again one can 
calculate $C_{N}$ if all sources ( $B^{\prime} S Q^{\ell / B p}$ ) are completely known. In practice, one tries to measure $C_{N}$, or at least its magnitude $\left|C_{N}\right|$, and find the coupling coefficient $k$ from that. Conversely, one can control $\kappa$ through $C_{N}$ by changing $\left({ }^{S} \mathrm{SQ}^{l / B p}\right)$ of the correction skew quadrupoles.

In the present design of 7-GeV ring, $\nu_{x}=35.216$ and $\nu_{y}=14.298$ so that $N=21$ (with minus sign for \pm ) and $\Delta=-0.082$. There will be twenty correction skew quadrupole magnets, one in every other cell, with $\left|B^{\prime} S Q^{\ell}\right|<0.3 \mathrm{~T}$.

II. Useful Relations

In this section, the fractional part of the tune is designated by $Q$ :

$$
v=\text { integer }+Q
$$

The integer part of $v$ must be measured so that there will be no ambiguities. In addition, it is usually necessary to determine whether $Q$ is above or below one-half. One convenient way to see this is to observe the closed orbit pattern created by a single kick.

It can be shown that

$$
\begin{gathered}
\Delta \equiv v_{x}-v_{y}-N=Q_{x}-Q_{y} \\
\mu \equiv Q_{1}-Q_{2}=\sqrt{\Delta^{2}+\left(2\left|C_{N}\right|\right)^{2}} \\
Q_{1}+Q_{2}=Q_{x}+Q_{y}
\end{gathered}
$$


It the emittance $\varepsilon_{x}=\varepsilon_{0}$ and $\varepsilon_{y}=0$ initially, there will

be an exchange of energy from one transverse direction to the other and both $\varepsilon_{x}$ and $\varepsilon_{y}$ will oscillate between two extreme values:

\begin{tabular}{|c|c|c|}
\hline & $\varepsilon_{X}$ & $\varepsilon_{y}$ \\
\hline $\max$ & $\varepsilon_{0}$ & $4\left|C_{N}\right|^{2} \cdot \varepsilon_{0} / \mu^{2}$ \\
\hline $\min$. & $o^{\Delta^{2} / \mu^{2}}$ & 0 \\
\hline
\end{tabular}

The period of this oscillation is $(1 / \mu)$ revolutions. This is usually much less than the damping time so that what one observes are the average over many revolutions:

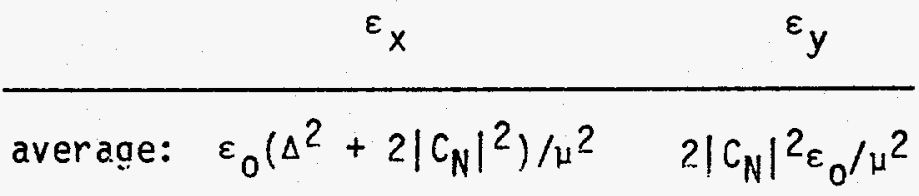

Note that $\varepsilon_{x}+\varepsilon_{y}=\varepsilon_{0}$ since the total energy must be conserved. [If the operating point is near a sum resonance, $\nu_{x}+\nu_{y}=$ integer $+\Delta$, $\left(\varepsilon_{x}-\varepsilon_{y}\right)$ instead of $\left(\varepsilon_{x}+\varepsilon_{y}\right)$ becomes a constant of the motion. There will be an increase in the total energy of transverse oscillations.] Comparing (II. 6) with (I. 1), one finds

$$
\kappa=2 \mid C_{N}^{2} /\left\{\Delta^{2}+2\left|C_{N}\right|^{2}\right\}
$$


Under the assumption that only one component $C_{N}$ is important in describing the linear coupled motion, a complete coupling $k=1$ is possible only with $\Delta=0$, i.e., $v_{x}-v_{y}=$ integer.

Based on a purely statistical argument, one can estimate the expected rms value of $\left|C_{N}\right|$ when the rms values of quadrupole roll $\left\langle\theta_{R}\right\rangle$ and of the vertical closed-orbit deviation $\left\langle y_{C}\right\rangle$ in sextupoles are known. Both $\theta_{R}$ and $y_{C}$ are assumed to be random. This is certainly the case for $\theta_{R}$. It is probably true for $y_{c}$ as well, if $y_{c}$ is the residual closed orbit after correction.

(1) quadrupole roll

$$
\left\langle\left|C_{N}\right|\right\rangle=(1 / 4 \pi) 2\left\langle\theta_{R}\right\rangle \sqrt{\sum\left(\beta_{x} \beta_{y}\right)\left(B^{\prime} \ell / B \rho\right)^{2}} \approx 15\left\langle\theta_{R}\right\rangle
$$

For $\left\langle\theta_{R}\right\rangle=0.2 \mathrm{mrad},\left\langle\left|C_{N}\right|\right\rangle=0.003$ which is much less than $|\Delta|=0.08$

(2) vertical closed orbit in sextupoles

$$
\left\langle\left|C_{N}\right|\right\rangle=(1 / 4 \pi)\left\langle y_{C}\right\rangle \sqrt{\sum\left(\beta_{x}^{\beta} y^{\prime}\right)\left(B^{\prime \prime} \ell / B \rho\right)^{2}} \approx 42 m^{-1}\left\langle y_{C}\right\rangle
$$

For $\left\langle y_{C}\right\rangle=0.2 \mathrm{~mm},\left\langle C_{N} \mid\right\rangle=0.008$. If two effects are combined in quadrature, $\left\langle\left|C_{N}\right|\right\rangle=0.0085$

The discussions presented here do not take into account the effective increase of $\varepsilon_{x}$ or $\varepsilon_{y}$ because of the finite momentum spread in the presence of momentum dispersions. If this effect is included, the simple 
relation between $\left|C_{N}\right|$ and $K,(I I, 7)$, is no longer valid. One can of course repeat the procedures for each value of $(\Delta p / p)$ and integrate the results. The virtue of simplicity will be lost, however, and it will be more practical to resort to numerical tracking studies.

III. Measurement of $\left|C_{N}\right| \cdot 4,5$

It is possible to measure $\left|C_{N}\right|$ by pinging the beam in one direction (usually horizontal) and then observing the oscillation patterns in both directions if oscillations can be sustained for a hundred revolutions or so. 5 During the commissioning of Tevatron at Fermilab, $\left|C_{N}\right|$ has been obtained together with two chromaticities in the following manner. Note that the identical procedures can be used for the measurement of $B_{x}$ and $B_{y}$ instead of chromaticities $\xi_{x}$ and $\xi_{y}$. All one must do is to use, in place of $\delta_{p}=(\Delta p / p)$, various values of $\left(B^{\prime} \ell / 4 \pi B \rho\right)$ of a special quadrupole located at a place where $\beta_{x}$ and $\beta_{y}$ are to be measured, and to use $\beta_{x}$ and $\beta_{y}$ in $p l a c e$ of $\xi_{x}$ and $\xi_{y}$, respectively.

In order to find chromaticities $\xi_{x}$ and $\xi_{y}$, one measures two eigentunes $\left(\nu_{1}\right.$ and $\left.v_{2}\right)$ as a function of $\delta_{p}=(\Delta p / p)$. The change in $\delta_{p}$ is introduced by the corresponding change in $\mathrm{rf}$ frequency $\Delta f / f$, since (for a fixed bend field)

$$
\delta_{p}=-\gamma_{t}^{2}(\Delta f / f)
$$


Suppose one obtained data such as the one shown below.

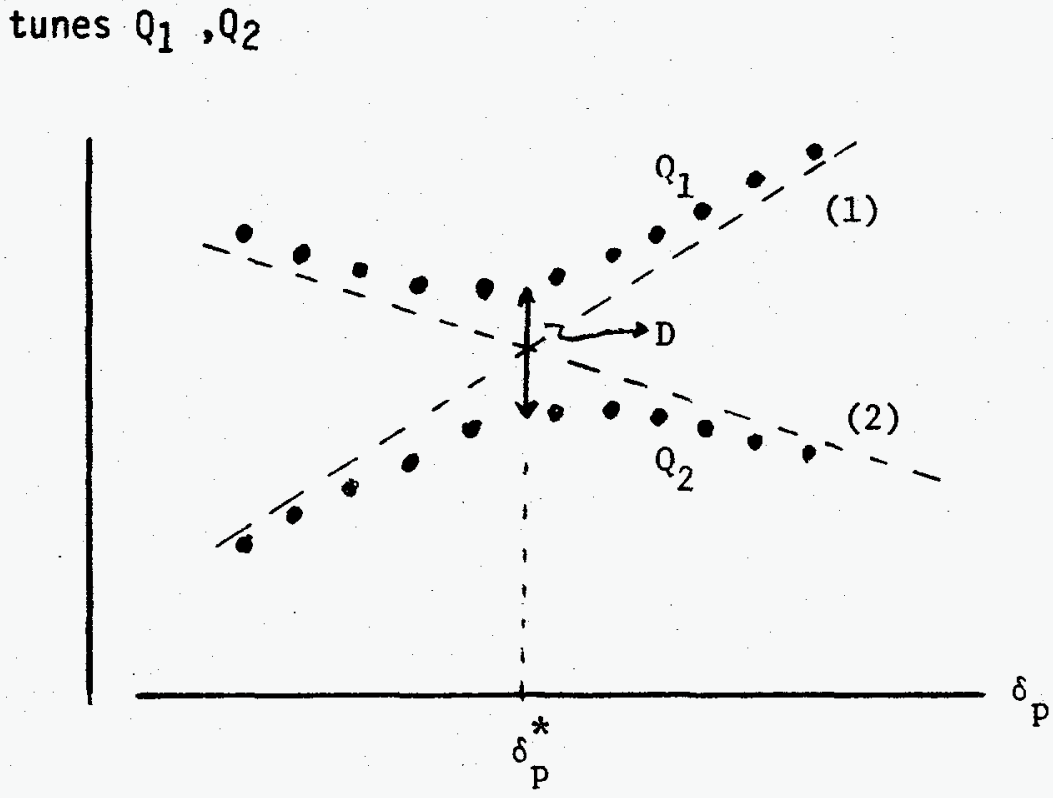

It is assumed here that the two frequencies $Q_{1}$ and $Q_{2}$ are measured so well that the data form a hyperbola. In real life, it is necessary to do some smoothing of data. As shown below, asymptotes (1) and (2) represent two chromaticities but "which is which" must be decided by the identification one can make at $\delta_{p}$ far away from $\delta_{p}{ }^{*}$. As one scans the tunes across $\delta_{p}$, the "horizontal" tune shifts from $Q_{1}$ (or $Q_{2}$ ) curve to $Q_{2}$ (or $Q_{1}$ ) curve. Near, it is often difficult to decide which is horizontal and which is vertical. This is understandable since the two frequencies are equally important in describing horizontal or vertical motion.

Two tunes $v_{x}=$ integer $+Q_{x}$ and $v_{y}=$ integer $+Q_{y}$ are what one would have in the absence of linear coupling. If one defines a number of parameters, 


$$
\begin{aligned}
& Q_{x}=Q_{x_{0}}+\xi_{x} \delta_{p}, Q_{y}=Q_{y_{0}}+\xi_{y} \delta_{p}, \\
& \bar{Q}=\left(Q_{1}+Q_{2}\right) / 2=\left(Q_{x}+Q_{y}\right) / 2=\bar{Q}_{0}+\bar{\xi}_{p}, \\
& \bar{\xi}=\left(\xi_{x}+\xi_{y}\right) / 2, \quad \Delta_{\xi}=\xi_{x}-\xi_{y}, \\
& \Delta=Q_{x}-Q_{y}=\Delta_{0}+\Delta_{\xi} \delta_{p}, \quad \Delta_{0}=Q_{x_{0}}-Q_{y_{0}}
\end{aligned}
$$

one finds from (II. 3) that

$$
\begin{array}{r}
Q_{1}+Q_{2}=2\left(\bar{Q}_{0}+\bar{\xi} \cdot \delta_{p}\right) \\
Q_{1}-Q_{2}=\sqrt{4\left|C_{N}\right|^{2}+\left(\Delta_{0}+\Delta_{\xi} \delta_{p}\right)^{2}}
\end{array}
$$

$Q_{1}$ and $Q_{2}$ as a function of $\delta_{p}$ are indeed on a hyperbola. Equations for two asymptotes are

$$
\left\{\bar{Q}_{0} \pm\left(\Delta_{0} \Delta_{\xi} / 2\left|\Delta_{\xi}\right|\right)\right\}+\left\{\xi \pm\left|\Delta_{\xi}\right| / 2\right\} \delta_{p}
$$

indicating that the slopes of them are two chromaticities $\xi_{x}$ and $\xi_{y}$. Furthermore, the shortest distance between two branches of hyperbola is a measure of the coupling parameter $\left|C_{N}\right|$ :

$$
\text { distance } D=2 \cdot\left|C_{N}\right|
$$


Least-squares analysis of data for $\left(Q_{1}+Q_{2}\right)$ will give two parameters $\bar{Q}_{0}$ and $\bar{\xi}$ while the analysis of $\left(Q_{1}-Q_{2}\right)$ will give the remaining three parameters $\Delta_{0}, \Delta \xi$ and $\left|C_{N}\right|$. Obviously, two chromaticities are given by $\left(\xi+\Delta_{\xi} / 2\right)$ and $\left(\xi-\Delta_{\xi} / 2\right)$.

\section{References}

1. D. A. Edwards and L. C. Teng, IEEE Trans. Nuc7. Sci. MS-20, 885 (1973)

2. E. D. Courant and H. S. Snyder, Annals of Physics 3, I (1958).

3. Matthew Sands, "The Physics of Electron Storage Rings - An Introduction," SLAC-121 (November 1970).

4. G. Guignard, CERN Report ISR-MA/75-23, PART I, (June 27, 1975).

5. S. Ohnuma, Fermilab Accelerator Experiment EXP-85 (September 14, 1977).

\section{DISCLAIMER}

This report was prepared as an account of work sponsored by an agency of the United States Government. Neither the United States Government nor any agency thereof, nor any of their employees, makes any warranty, express or implied, or assumes any legal liability or responsibility for the accuracy, completeness, or usefulness of any information, apparatus, product, or process disclosed, or represents that its use would not infringe privately owned rights. Reference herein to any specific commercial product, process, or service by trade name, trademark, manufacturer, or otherwise does not necessarily constitute or imply its endorsement, recommendation, or favoring by the United States Government or any agency thereof. The views and opinions of authors expressed herein do not necessarily state or reflect those of the United States Government or any agency thereof. 